\title{
Role of Magnetic Resonance Imaging in Evaluation of Adnexal Pathologies and Correlation with Sonography
}

\author{
Shiva Shankar M P1, Venkateshwaran K N², Gokulakrishnan R M³ ${ }^{3}$, Sinduja Paul ${ }^{4}$, Balaji Ramraj ${ }^{5}$ \\ ${ }^{1}$ Assistant Professor, Department of Radiodiagnosis, SRM Medical College Hospital and Research Centre, Kattankulathur, \\ Kancheeepuram, Tamilnadu, ${ }^{2}$ Assistant Professor, Department of Radiodiagnosis, SRM Medical College Hospital and Research \\ Centre, Kattankulathur, Kancheeepuram, Tamilnadu, ${ }^{3}$ Junior Resident, Department of Radiodiagnosis, SRM Medical College \\ Hospital and Research Centre, Kattankulathur, Kancheeepuram, Tamilnadu, ${ }^{4}$ Junior Resident, Department of Radiodiagnosis, \\ SRM Medical College Hospital and Research Centre, Kattankulathur, Kancheeepuram, Tamilnadu, ${ }^{5}$ Associate Professor, \\ Department of Community Medicine, SRM Medical College Hospital and Research Centre, Kattankulathur, Kancheeepuram, \\ Tamilnadu, India
}

Corresponding author: Dr Venkateshwaran K N, Assistant Professor, Department of Radiodiagnosis, SRM Medical College Hospital and Research Centre, Kattankulathur, Kancheeepuram, Tamilnadu, India

DOI: http://dx.doi.org/10.21276/ijcmsr.2019.4.2.2

How to cite this article: Shiva Shankar M P, Venkateshwaran K N, Gokulakrishnan R M, Sinduja Paul, Balaji Ramraj. Role of magnetic resonance imaging in evaluation of adnexal pathologies and correlation with sonography. International Journal of Contemporary Medicine Surgery and Radiology. 2019;4(2):B6-B10.

\section{A B S T R A C T}

Introduction: Adnexal masses pose a diagnostic dilemma to the gynecologist as well as radiologist because of their varied spectrum. The most important thing that needs to be determined is that whether the lesion is benign or malignant, so that the patient gets the appropriate treatment based on the pathology. The two important modalities widely used for diagnosis of adnexal pathologies are ultrasound and magnetic resonance imaging [MRI]. Study aimed to compare MRI and ultrasound for identification of organ of origin and characterization of adnexal lesions.

Material and methods: A prospective study was done on 82 patients who were referred to radiology department with suspected adnexal pathologies. All patients were subjected to ultrasound and MRI examination and final correlation with histopathology was done. The agreement between these modalities and histopathology/ final diagnosis was calculated. The sensitivity and specificity of these modalities to correctly distinguish benign and malignant pathologies was calculated. Results: Among 82 patients, the origin of lesion was ovarian in $68(83 \%)$, extra ovarian in $5(6 \%)$, fallopian tube in $8(10 \%)$ and broad ligament in 1(1\%) patient in MRI. 52 (63\%) were purely cystic, $14(17 \%)$ were solid and $16(20 \%)$ were complex in MRI. There was excellent agreement between MRI and final diagnosis in both origin and characterization of mass ( $\mathrm{k}$ value $>0.9$ ). The sensitivity, specificity and accuracy for diagnosing malignant lesions by MRI were $97.1 \%, 100 \%$ and $97.5 \%$ respectively. Conclusion: Ultrasound can be used as initial investigation for screening pelvic masses and for follow up. MRI is superior to ultrasound in identifying both origin and characterization of adnexal masses. It is also found to be of great importance in differentiating between benign and malignant pathologies. This will definitely help in avoiding unnecessary surgeries in case of benign pathologies and will be of great help in planning treatment options for malignant pathologies.

Keywords: Magnetic Resonance Imaging, Ultrasound, Adnexal Pathologies, Fallopian Tube, Broad Ligament

\section{INTRODUCTION}

Adnexal pathologies are commonly encountered among women of all age groups. These adnexal lesions usually present with varied range of spectrum which can be benign masses like functional cysts or malignant masses like ovarian cancer. The benign lesions are usually asymptomatic and most of times doesn't need any treatment and can be just followed up. On the other hand malignant lesions usually need proper diagnosis and treatment.

Adnexal masses usually pose a challenging situation to the treating gynecologist as well as radiologist because of the differential diagnosis which is often broad and complex. The most important thing that needs to be determined is that whether the lesion is benign or malignant, so that the patient gets the appropriate treatment based on the pathology. Determining the benign nature of the mass will save the patient from further investigation and unnecessary surgery and malignant masses need to be identified as early as possible so that the patient gets the early and appropriate treatment.

The two important modalities widely used for diagnosis of adnexal pathologies are ultrasound and magnetic resonance imaging. The advantages of ultrasound are easy availability and simplicity of the examination. However, the drawbacks include obscuration of adnexa by bowel gas, limited field of view, and its huge dependence on the skill of the radiologists. ${ }^{1}$ Around 20\% of adnexal pathologies cannot be diagnosed correctly in sonography as organ of origin cannot be assessed properly nor its classification of a lesion into benign and 
malignant lesion cannot be done accurately. Even though sonography has high sensitivity for the detection of masses, it has decreased specificity for characterization of the masses, which usually ranges from $60 \%$ to $95 \%$ in previous studies. ${ }^{2}$ Magnetic resonance imaging with its high resolution and multi planar imaging has the ability to characterize adnexal lesions accurately and currently the modality of choice. ${ }^{3}$ The soft tissue differentiation characteristics of MRI are also enhanced by using various pulse sequences and contrast studies which results in higher sensitivity and specificity.

MR is usually considered as a next step in the evaluation of a lesion after sonography. The main drawback of MRI lies in it not being readily available and expensive compared to ultrasound. It also is non advisable for patients with certain metallic implants and claustrophobic patients. Gynecologists referring cases and the general radiologists are mostly in confusion in finding out the appropriate patients needing an MRI examination for assessment of adnexal pathologies.

Study aimed to compare MRI and ultrasound in detection and identification of organ of origin and characterization of adnexal lesions and to compare MR and ultrasound in distinguishing benign and malignant adnexal lesions accurately.

\section{MATERIAL AND METHODS}

The study was conducted after getting approval from our institution's ethical committee and after obtaining written informed consent from the patients. The study was conducted from period of January 2016 to August 2017 in Department of Radiology, SRM medical college hospital and Research centre, Kattankulathur, Kanchipuram district. A prospective study was done on 82 patients who were referred to radiology department with suspected adnexal pathologies. All patients were subjected to ultrasound and MRI examination if adnexal pathology is found in ultrasound. Final correlation with histopathology was done if available.

Ultrasound imaging was performed using GE logic F8 ultrasound machine. Transabdominal ultrasound was done using a probe of $3.5-5 \mathrm{MHz}$ and transvaginal ultrasound was done using a probe of $10 \mathrm{MHz}$. Transabdominal ultrasound was done with full bladder with optimal settings. Transvaginal ultrasound was done with an empty bladder. The following findings were noted in ultrasound examination including ovarian size and echo texture, bilateral adnexa, and fallopian tubes. If adnexal mass was seen number, size, origin of mass (ovarian, uterine, or extra ovarian), and characterization (solid, cystic, complex solid cystic) were noted. The details about uterus and cervix along with any lesion if present were also noted.

MRI was performed using 1.5 Tesla Siemens Magnetome Essenza machine. The following sequences were done including T1 WI, T1 WI fat saturation, T2 WI, T2WI fat saturation and STIR in axial plane, T2 WI fat saturation and STIR in coronal plane and T2 WI in sagittal plane. Contrast and other special sequences like diffusion and gradient imaging were used as and when required. Apart from findings mentioned in ultrasound, signal characteristics of lesions in both T1 and T2W were noted for presence of fat, hemorrhage, fluid and solid components. Extent of lesion was noted in case of ovarian carcinoma along with presence of peritoneal deposits, lymph nodes and ascites. The findings including thick enhancing wall, solid lesion enhancement, thick enhancing or non-enhancing septations, and mural nodules were used to characterize a mass as malignant.

\section{Inclusion criteria}

All patients referred to the department of Radiology with clinically suspected pelvic lesions and found to have adnexal pathology in Ultrasound. The patients in whom subsequently MRI was done were included in the study.

\section{Exclusion criteria}

Subjects with metallic fixations or with cardiac pacemakers. Claustrophobic patients.

All patients where surgery was not done or lost to follow up.

\section{STATISTICAL ANALYSIS}

The data was analyzed by using SPSS software version 19.0. Descriptive analysis such as frequency, percentage were used to describe the data and inferential statistics such as chi-square test, sensitivity, specificity, accuracy, positive and negative predictive value were used to analyze the data. The agreement between two imaging modalities with final diagnosis with reference to origin and characterization of lesion was established using the kappa statistics.

\section{RESULTS}

A total of 82 participants were enrolled in the study. The majority of the patients were in the age group $21-40$ years - 27 patients $(32 \%)$ and followed by $1-20$ years -23 patients (28\%). Majority of them were in pre menopause period 61 (74\%) and the rest in the post menopause period 21 (26\%). Among them 62 (76\%) suffered with pain, 22 (26\%) suffered with lump 20(24\%) suffered with abnormal bleeding and 18 (21\%) were suffering from irregular periods. The laterality of lesion was on right side 35 (43\%), left side 37 (45\%) and bilateral $10(12 \%)$ patients.

The origin of lesion was found to be ovarian in $72(87 \%)$,

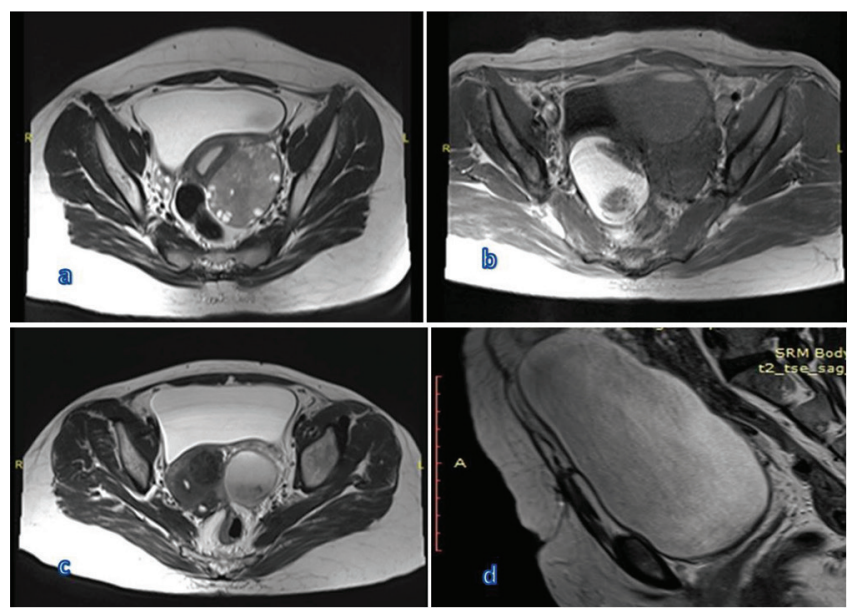

Figure-1: (a) Axial T2 WI showing enlarged left ovary with peripherally arranged follicles c/w torsion (b) Axial T1 WI showing right $\mathrm{T} 1$ hyperintense adnexal cyst c/w dermoid cyst. (c) Axial T2WI showing left adnexal cyst with shading sign c/w endometrioma (d) Sagittal T2WI showing large clear adnexal cystic lesion c/w benign cystic neoplasm. 


\begin{tabular}{|l|c|c|c|}
\hline Origin of lesion & Ultrasound & MRI & Final/HPE diagnosis \\
\hline Ovary & 72 & 68 & 66 \\
\hline Extra ovarian & 3 & 5 & 6 \\
\hline Fallopian tube & 6 & 8 & 8 \\
\hline Broad ligament & 1 & 1 & 2 \\
\hline Agreement of ultrasound with final diagnosis: $\mathrm{k}-0.742 \mathrm{Cl}$ \\
- 0.643 to $0.841, \mathrm{p}-0.0001 ;$ Agreement of MRI with final \\
diagnosis: $\mathrm{k}-0.923, \mathrm{Cl}-0.870$ to $0.976, \mathrm{p}-0.0001$ \\
\hline \multicolumn{4}{|c|}{ Table-1: Origin of lesions in ultrasound/ MRI/ HPE } \\
\hline
\end{tabular}

\begin{tabular}{|l|c|c|c|}
\hline & Ultrasound & MR & Final/ HPE diagnosis \\
\hline CYSTIC & 42 & 52 & 53 \\
\hline Solid & 16 & 14 & 14 \\
\hline Complex & 24 & 16 & 15 \\
\hline
\end{tabular}

Agreement of ultrasound with final diagnosis: $\mathrm{k}-0.770, \mathrm{Cl}$ -0.706 to $0.834, p-0.0001$; Agreement of MRI with final diagnosis: $\mathrm{k}-0.977, \mathrm{Cl}-0.954$ to $1.00, \mathrm{p}-0.0001$

Table-2: Characterization of lesions in ultrasound/ MRI/ HPE

\begin{tabular}{|l|c|c|}
\hline & Ultrasound & MRI \\
\hline Simple ovarian cyst & 8 & 6 \\
\hline Para-ovarian cyst & 4 & 6 \\
\hline Hemorrhagic cyst & 10 & 12 \\
\hline Endometriotic cyst & 8 & 12 \\
\hline Hydrosalphinx & 6 & 8 \\
\hline Dermoid cyst & 2 & 4 \\
\hline Benign solid neoplasm & 2 & 3 \\
\hline Torsion ovary & 8 & 8 \\
\hline Ectopic pregnancy & 2 & 2 \\
\hline Broad ligament fibroid & 1 & 1 \\
\hline Benign cystic neoplasm & 4 & 4 \\
\hline Malignant mass & 6 & 8 \\
\hline Indeterminate nature & 21 & 8 \\
\hline \multicolumn{2}{|c|}{ Table-3: Distribution of lesions in ultrasound and MRI } \\
\hline
\end{tabular}

extra-ovarian in 3(4\%), fallopian tube in 6(8\%) and broad ligament in $1(1 \%)$ patient on ultrasound. In MRI the origin of lesion was ovarian in 68(83\%), extra ovarian in 5(6\%), fallopian tube in $8(10 \%)$ and broad ligament in $1(1 \%)$ patient. Two cases of extra ovarian lesions (para ovarian and para tubal cysts) and two cases of hydrosalphinx were misdiagnosed as ovarian cysts in ultrasound. The origin of lesion in final / HPE diagnosis was similar to MRI except for two patients where one case of peritoneal inclusion cyst and broad ligament fibroid were misdiagnosed as ovarian lesions in MRI. The agreement of ultrasound with final diagnosis for detection of origin of lesion was good (k-0.742) and agreement of MRI with final diagnosis was excellent ( $k$ 0.923). The origin of lesion in ultrasound and MRI and correlation with final diagnosis is illustrated in Table1.

On Ultrasound, 42 (51\%) of the adnexal masses were purely cystic, 16 (20\%) were solid and 24 (29\%) were complex. On MRI, 52 (63\%) were purely cystic, 14 (17\%) were solid and $16(20 \%)$ were complex. The final diagnosis of lesions was similar to MR except for one case of endometriotic cyst which was misdiagnosed as complex cyst in MRI. The agreement of ultrasound with final diagnosis for detection of origin of lesion was good (k-0.770) and agreement of MRI with final diagnosis was excellent $(\mathrm{k}-0.977)$. The characterization of lesion in ultrasound and MRI and correlation with final diagnosis is illustrated in Table-2.The distribution of lesions in ultrasound and MRI is illustrated in Table-3.

Among 82 cases, 50 cases underwent surgical procedures and histopathological diagnosis was obtained. The remaining 32 cases were managed conservatively and radiological and clinical follow up was done for a year. All those 32 cases which were not operated had classical imaging findings and no significant change was seen or regression of lesion was seen on follow-up which was convincing enough to make a definite diagnosis radiologicaly. Out of 50 cases the lesions were classified as benign, probably benign, malignant and probably malignant in both ultrasound and MRI. For statistical purpose the lesions were divided as either benign or malignant in both ultrasound and MRI and compared with histopathological diagnosis. The sensitivity, specificity, accuracy, positive and negative predictive value was calculated for both the modalities to correctly classify the lesion as either benign or malignant.

Out of 50 lesions, 14 lesions turned out to be malignant in histopathological diagnosis. Out of 50 lesions 16 lesions were classified as malignant by MRI and 19 lesions as malignant by ultrasound. As such two lesions in MRI and five lesions in ultrasound are over-diagnosed as malignant lesions which turned out to be benign on histopathology. One lesion over diagnosed in MRI was case of broad ligament fibroid which was misdiagnosed as solid ovarian tumor. Another was case of ovarian cystadenoma which was classified as malignant in MRI due to mildly thickened wall and thickened septa. In addition a case of ovarian carcinoma was misdiagnosed as benign cystic neoplasm in ultrasound. The sensitivity, specificity, accuracy, positive and negative predictive value for correctly distinguishing benign and malignant pathologies in ultrasound and MRI was 92.5\%, 93.3\%, 92.7\%, 98.4\%, $73.7 \%$ and $97.1 \%, 100 \%, 97.5 \%, 100 \%, 87.5 \%$.

\section{DISCUSSION}

The most important step in evaluation of adnexal masses is to determine whether the mass is benign or malignant as it has important role in treatment procedure and to decide whether patient needs surgery. In our study 82 female patients with adnexal masses were studied by ultrasound and MRI modalities. Among 82 cases, 50 cases underwent surgical procedures and histopathological diagnosis was obtained. The remaining 32 cases were managed conservatively and radiological and clinical follow up was done for a year. In our present study, the mean age was 32 years and the most commonly affected age group was $21-40$ years. This was similar to other studies done by Aruna et $\mathrm{al}^{4}$ where mean age was 30 years and Al-Shukri et $\mathrm{al}^{5} 29$ years. However the mean age was much higher 46 years in Adusumilli et al. ${ }^{6}$ Benign cysts are much more common in reproductive age group, while the malignant lesions are more common in the postmenopausal age group. In our study predominant pathologies are benign lesions and hence mean age was lesser. In our study, the most common presenting complaints were lower abdominal pain in $76 \%$ cases and pelvic lump in $26 \%$ cases. Our findings are 
similar to those of Guzel $\mathrm{Al}$ et $\mathrm{al}^{7}$ where the predominant compliant was abdominal pain in $77.5 \%$ cases and study by Al-Shukri et $\mathrm{al}^{5}$ where the presenting symptom was of lower abdominal pain in $98 \%$ cases. Regarding anatomical site of adnexal masses, in our study maximum number of cases $(80 \%)$ was seen arising from the ovaries and in study by Aruna et $\mathrm{al}^{4} 68 \%$ of cases were seen arising from ovary. In study by Adusumilli et $\mathrm{al}^{6}$ ovarian masses were found to be most common (56\%) of all the adnexal masses where they included significant number of leiomyomas. In our study only 2 broad ligament fibroids were included and most of the pathologies are ovarian with little number of pathologies in fallopian tube and extra ovarian.

The three most important things that were noted for assessment of adnexal masses in our study were 1 . Origin of mass 2. Characterization of mass 3. Classification of mass as either benign or malignant.

The first step in diagnosis of adnexal mass is to ascertain the origin of mass. In our study there was excellent agreement seen between MRI and the origin of a mass. In our study the origin of mass was wrongly interpreted in MR only in two cases. One was a large broad ligament fibroid which was misinterpreted as solid ovarian tumor and another was peritoneal inclusion cyst which was interpreted as ovarian cyst. In both cases the mistake could have been avoided if normal separate ovary could have been visualised. But however they were not visualized separately as the ovary was smaller and was seen displaced by the larger lesion. The signal intensity of fibroid is exactly similar (T2 hypointense solid nature) to solid ovarian tumor which made the diagnosis difficult. The morphology of peritoneal inclusion cyst is similar to ovarian cyst making the diagnosis difficult.

On the other hand ultrasound had only good agreement for determining the origin of the mass. Two cases of hydro/ hematosalphinx were misdiagnosed as ovarian lesion as ovary was not separately visualised and the incomplete septae in dilated fallopian tube was misdiagnosed as ovarian lesion with septae. Similarly three para-ovarian lesions including para-ovarian cyst, peritoneal inclusion cyst and paratubal cyst were misdiagnosed as ovarian lesions as ovary was not seen separately and as both of them look similar sonologically. One case of broad ligament fibroid was misdiagnosed as solid ovarian tumor similar to MRI.

Ofer Benjaminov et $\mathrm{al}^{8}$ in their study of ultrasound of the fallopian tube pathologies had explained that identification of ovary separate from the lesion will help in differentiating hydrosalphinx from a complex ovarian mass. In some cases, severely scarred hydrosalphinx may mimic complex ovarian lesion. They suggested that the pitfalls in the diagnosis of hydrosalpinx included paratubal, paraovarian, or perineural cysts and these may be better delineated in MRI.

The agreement with MRI was similar to study by Adusumilli et $\mathrm{al}^{6}$ where $\mathrm{k}$ value was 0.93 however with ultrasound there was significantly increased agreement in our study compared to them ( $\mathrm{k}$ value 0.742 in our study compared to 0.19 by Adusumilli et al).

The second most important step is characterization of mass. In our study MRI had excellent agreement whereas ultrasound had good agreement. In the study by Prabha et $\mathrm{al}^{9}$ they found
0\%, 66\% and 42\% lesions on USG to be cystic, solid and complex respectively, whereas, the MRI showed 27\%, 37\% and $31 \%$, cystic, solid and complex lesions respectively. In study by Aruna et $\mathrm{al}^{4}$ on USG, $50 \%$ of the adnexal masses were cystic, $18 \%$ were solid and $32 \%$ were complex. On MRI, $56 \%$ were cystic, $18 \%$ were solid and $26 \%$ were complex which is similar to our study. The agreement with MRI was similar to study by Adusumilli et $\mathrm{al}^{6}$ where $\mathrm{k}$ value was 0.93 however with ultrasound there was significantly increased agreement in our study compared to them ( $\mathrm{k}$ value 0.770 in our study compared to 0.33 by Adusumilli et al).

MRI correctly revealed the tissue content of all masses except one case of endometriotic cyst which was diagnosed as complex adnexal mass in MR which was found to be completely cystic post surgery. MR with excellent soft tissue characterization properties helps in identifying specific tissue characteristics such as fat, hemorrhage, fluid and fibrous tissue. Ultrasound on the other hand had less agreement compared to MR. Few cases of hemorrhagic cyst, endometrioma and dermoid were misdiagnosed as complex adnexal masses due to their varied appearance in ultrasound.

Douglas et $\mathrm{al}^{10}$ in their study of characterization of adnexal lesions through ultrasound stated that endometriotic cysts can have a diffuse appearance and similar findings via sonography can overlap in other lesions like haemorrhagic cysts, dermoid cysts and other lesions. Sugimura et al ${ }^{11}$ stated in his study of multimodality imaging of ovarian cystic lesions that sonographically, endometriotic lesions can have a varied appearance and on MRI it can be revealed whether it contains blood products or not with the high signal intensity on $\mathrm{T} 1$ weighted images and intermediate to low signal intensity on T2 weighted images.

Corwin et $\mathrm{al}^{12}$ did a study on differentiation of ovarian endometriomas from haemorrhagic cysts on MR imaging based on T2 dark spot sign and finally concluded that T2 dark spot sign has high specificity for chronic haemorrhage and is useful in differentiating endometriomas from haemorrhagic cysts. Shading sign in T2 weighted images is sensitive but not specific in case of endometriomas. In our study, two patients with endometrioma showed a T2 darkspot sign whereas shading sign was seen in 8 of the endometriotic cysts. Two patients of hemorrhagic cyst showed shading sign whereas none of them showed dark spot sign.

Non contrast T1- and T2-weighted images are important for accurate tissue characterization. The presence of fat and blood are easily diagnosed on T1-weighted images and chronic blood degradation products and fibrous tissue in T2weighted images.

The third important step is the ability to say whether the lesion is benign or malignant. Guerra et $\mathrm{al}^{13}$ observed in their study of 161 patients that MRI had high sensitivity, specificity and accuracy of $98 \%, 93 \%$ and $95 \%$ to differentiate between benign and malignant adnexal lesions. Dodge et $\mathrm{al}^{14}$ in meta analysis found that the sensitivity and specificity of MRI for diagnosis of malignancy can reach $92 \%$ and $88 \%$, respectively. Aruna et $\mathrm{al}^{4}$ observed that MR imaging had a sensitivity of $100 \%$ and specificity of $97.7 \%$ whereas ultrasound had a sensitivity of $80 \%$ and specificity of $95 \%$. In a study done by Sohaib et $\mathrm{al}^{15}$ accuracy of MR imaging 
in the detection and characterization of adnexal mass lesions was reported to have a sensitivity of $95 \%$ and specificity of $88 \%$. The sensitivity and specificity of gray scale ultrasound in adnexal masses in a study conducted by Madan et $\mathrm{al}^{16}$ was $92.5 \%$ and $55.3 \%$. Similar results were given by Scoutt et $\mathrm{al}^{17}$ and Hriack et al. ${ }^{18}$ The sensitivity and specificity of MRI in our study was 97.1 and 100\% which was similar to above studies. The sensitivity and specificity of ultrasound was $92.5 \%$ and $93.3 \%$ which is slightly lesser to MRI.

\section{CONCLUSION}

Adnexal masses are commonly encountered pathologies in the reproductive age group and are most often of ovarian origin. Even though ultrasound is used as screening tool for diagnosing pelvic masses, MRI is superior to ultrasound in identifying origin characterization and benignity of adnexal lesions. Still we realized there were few pitfalls in diagnosing adnexal masses in MRI. The first was in characterization of large clear cystic lesion. MRI could not categorize as lesion is whether it is simple ovarian/ adnexal cyst or benign cystic neoplasm as both of them could be unilocular, completely cystic and without any other specific characteristics. The second was in characterization of cystic mass with intermediate wall and septa thickness without any ascites or other features of malignancy. Both benign and malignant cystic neoplasm could present in this way and hence MRI could not completely rule out malignancy in such cases. The third scenario is in case of solid enhancing T2 hypointense adnexal mass. Both malignant solid ovarian lesions and benign pathologies including fibroid and benign solid ovarian fibrous lesions can present in this way and hence MRI can't completely avoid surgery in such cases.

\section{REFERENCES}

1. Smorgick N, Maymon R. Assessment of adnexal masses using ultrasound: a practical review. Int J Womens Health. 2014; 6(1): 857-863.

2. Funt SA, Hann LE. Detection and characterization of adnexal masses. Radiol Clin North Am. 2002; 40(3): 591-608.

3. Yamashita Y, Hatanaka Y, Torashima M, Takahashi M, Miyazaki K, Okamura H. Characterization of sonographically indeterminate ovarian tumors with MR imaging: a logistic regression analysis. Acta Radiol. 1997; 38(4):572-577.

4. Aruna K, Sumana C. Diagnosis of Adnexal Masses Using Ultrasound and Magnetic Resonance Imaging for Proper Management. Asian Pac. J. Health Sci. 2016; 3 (4):279-284.

5. Al-Shukri M, Mathew M, Al-Gafri W et al. A Clinicopathological Study of Women with Adnexal Masses Presenting with Acute Symptoms. Ann Med Health Sci Res. 2014; 4(2): 286-288.

6. Adusumilli S, Hussain HK, Caoili EM, Weadock WJ. et al: MRI of Sonographically Indeterminate Adnexal Masses. American journal of roentgenology. 2006; 187(3): 732-740.

7. Guzel A1, Kuyumcuoglu U, Erdemoglu M. Adnexal masses in postmenopausal and reproductive age women. J ExpTherOncol. 2011; 9(2):167-9.
8. Ofer B, Mostafa A: Sonography of the Abnormal Fallopian Tube. American Journal of Roentgenology. 2004; 183(3): 737-742.

9. Prabha T, Goyal S, Mishra HK, Aggarwal A. Role of MRI in Evaluation of Female Pelvic Masses in Comparison to Ultrasonography. Journal of Evolution of Medical and Dental Sciences 2014; 3(59):13330-34.

10. Brown DL: A practical approach to the ultrasound characterization of adnexal masses. Ultrasound Q.2007; 23(2): 87-105.

11. Sugimura K, Okizuka N, Imaoka J, Kaji Y, Takahashi K et al: Pelvic endometriosis detection and diagnosis with chemical shift MR imaging. Radiology 1993; 188 (2); 435-8.

12. Corwin MT, Gerscovich EO, Lamba R, Wilson M, McGahan JP: Differentiation of ovarian endometriomas from hemorrhagic cysts at MR imaging: utility of the T2 dark spot sign. Radiology. 2014; 271(1):126-32.

13. Guerra A, Cunha T.M, Felix A, Magnetic Resonance Evaluation of Adnexal Masses Acta Radiologica. 2006; 49(6):700-9.

14. Dodge JE, Covens AL, Lacchetti C, et al. Preoperative identification of a suspicious adnexal mass: a systematic review and meta-analysis. Gynecol Oncol. 2012;126(1):157-167.

15. Sohaib SA, Mills TD, Sahdev A, et al. The role of magnetic resonance imaging and ultrasound in patients with adnexal masses. ClinRadiol. 2005; 60: 340-8.

16. Madan R, Narula MK, Chitra R, Bajaj P. Sonomorphological and color doppler flow imaging evaluation of adnexal masses. Indian J Radiol Imaging. 2004;14(2): 365-72.

17. Hricak H, Chen M, Coakley FV, Kinkel K, Yu K, Sica $\mathrm{G}$, et al. Complex adnexal masses: detection and characterization with MR imaging - multivariate analysis. Radiology 2000; 214(1): 39-46.

18. Scoutt LM, McCarthy SM, Lange R, Bourque A, Schwartz PE. MR evaluation of clinically suspected adnexal masses. J Comput Assist Tomogr. 1994; 18(3):609-618.

Source of Support: Nil; Conflict of Interest: None

Submitted: 16-01-2019; Accepted: 15-04-2019; Published online: 27-04-2019 